\title{
PRECESSION AND STELLAR KINEMATIC PARAMETERS FROM THE PROPER MOTIONS OF THE AGK3U
}

\author{
Wei Xue, Jing Wenjin, Xn Tong(pi \\ Shanghai (O)servatory, Chinese Academia of Sciences \\ 80 Nandan Road \\ Shanghai 200030 \\ CHINA
}

\begin{abstract}
Precessional corrections and stellar kinematic parameters have been derived from the proper motions of 142,171 stars in the updated version of AGK3 (AGK3U). The sky has been divided into 1332 small areas, in which mean proper motions for each component are formed for different magnitude intervals, with and withont exclusion of fast stars. Solutions were performed for various kinds of the mean proper motions. Maximum-likelihood algorithun is used to take into account the ellipsoirlal distribution of residual velocities. The results for precessional correction are, $\left.\Delta n=0^{\prime \prime} .47 \pm 0^{\prime \prime} .03 / \mathrm{cy}, \Delta l=-0\right)^{\prime \prime} .35 \pm 00^{\prime \prime} .02 / \mathrm{cy}$, and the results for Oort's constants are, $\mathrm{A}=9.5 \pm 1.5 \mathrm{~km} / \mathrm{s} / \mathrm{pc}, \mathrm{B}=-7.5 \pm 1.5 \mathrm{~km} / \mathrm{s} / \mathrm{pc}$.
\end{abstract}

\section{INTRODUCTION}

From the proper motions of the preliminary version of AGK3, Dieckvoss(106 7 ) has obtained estimates for precessional corrections $\Delta n=0^{\prime \prime} .51 \pm 0^{\prime \prime} .01 / \mathrm{cy}, \Delta k=-0^{\prime \prime} .32 \pm 0^{\prime \prime} .01 / \mathrm{cy}$, and galactic rotation constants $A=14.8 \pm 0.6 \mathrm{~km} / \mathrm{s} / \mathrm{kpc}, B=-11.3 \pm 0.6 \mathrm{~km} / \mathrm{s} / \mathrm{kp}, \mathrm{c}$. Asteriadlis(10TT) has determined the four quantities $\Delta n=00^{\prime \prime} .44 \pm 00^{\prime \prime} .02 / \mathrm{cy}, \Delta k=-00^{\prime \prime} .36 \pm()^{\prime \prime} .02$ $/ \mathrm{cy}, A=16.1 \pm 1.9 \mathrm{~km} / \mathrm{s} / \mathrm{kpc}, B=-9.0 \pm 1.9 \mathrm{~km} / \mathrm{s} / \mathrm{kpc}$, from the published AGK 3 proper motions.

In this investigation, we aimed to dorive the precessional corrections and the constants of galactic rotation on the basis of the AGK3U proper motions. In addition to the improvement of the proper motions, in the present work, we employed a new reduction method, namcly maxminum-likelihood algrithm, which takes into acconnt both the ellipsoidal clistribution of residual velocities and observational errors(Wei, 1987).

\section{THE MATERIAL}

AGI3U is an updated version of AGK3, which improved the AGK3 positions and proper motions using the observations of the Palomar '(Quick V' survey made for the construction of Hubble Space Tclescope Guide Star Catalogue(Bucciarelli et al, 1092). It provides now positions and proper motions for 170,464 stars north of -2.5 degree declination to the limiting magnitude 13.3 on magnetic tape. The positions have a mean error of $0^{\prime \prime} .16 \tau$ at an 
avcrage epoch of 1950.62 and the proper motions have a two dimensional mean error of $0^{\prime \prime} .82 / \mathrm{cy}$.

For each star the magnetic tape contains, besides the usual information (position, proper motion, magnitude, spectral type etc.), an error flag to indicate if linear model for determination of proper motion failed. Of the 170,464 AGK3U stars, we rejected 15,745 stars with an error flag, 5,735 OB stars and 6,813 stars brighter than $8^{\prime \prime \prime}$.

\section{DIVISION OF THE SKY AND FORMATION OF NORMAL PROPER MOTIONS}

The sky has been divided into 1332 areas of $4^{\circ} \times 4^{\circ}$ and normal proper motions formed within the areas, so that the computations can he manle more economically. The loss of accuracy in the final results caused by such a division is negligible, according to Asteri$\operatorname{ardis}(19 \pi T)$.

There are many stars with large proper motions in AGK3U. More than one tenth of the stars have a total proper motion larger than $6^{\prime \prime}$ per centery. The percentage of large motions decrease with magnitude. ( 16 percent in magnitude interval $8.0 \leq m_{p g}<0.0$, while 6 percent at $m_{p g} \geq 11.0$. In order to examine the effect of large proper motions in the solutions, we have formed the normal proper motion in the following ways:

$1, \mu<6^{\prime \prime} / \mathrm{cy}$, where $\mu=\left(\left(\mu_{c r} \cos \delta\right)^{2}+\mu_{\delta}^{2}\right)^{1 / 2}$

$2, \mu<12^{\prime \prime} / \mathrm{cy}$

3 , all the stars.

Slotions have been performed for the cases in different magnitude intervals and the whole material.

\section{NUMERICAL METHOD}

In the classical analyses of proper motions by the least square method, one has to assume that the combination of olsservational error and peculiar velocity can be treated as a single variable. In the straightforward case of analyses of a single component, the variance to be assigned to a star should depend on its position due to the assumed ellipsoidal distribution of peculiar velocities even if the observational error is the same for all of the stars. In the case of a combined solution of proper motions of two coordinates, not only will the peculiar velocity components depend on the position, but they will also be correlated(Murray, 1983). In order to overcome this difficulty, a maximum-likelihood algorithm has been de$\operatorname{veloperl}($ Wei, 198T).

It is found empirically that the axes of the velocity ellipsoid are almost the same as the Galactic-coordinate axes(Milablas and Binney, 1082), so it is reasonable to assume that the Galactic components of the residual $V\left(v_{1}, v_{2}, v_{3}\right)$ for an individual stars are sample from Gussian clistributions with zero means and variance $\sigma_{i}^{2}$, and they are independent of each other.

Let $\Delta\left(\Delta_{1}, \Delta_{2}\right)$ be the difference between the observed proper motions and expected proper notions, then such residnals can be expressed in the form of a linear function of $n_{i}$ : 


$$
\Delta_{j}=\sum_{i=1}^{3} a_{i j} V_{i}
$$

It is casy to shown that $\Delta_{1}, \Delta_{2}$ must be sampled from a gencral bivariate Ganssian distribution, and hence for all stars the likelihood function $L$ is of all of the peculiar velocity components obtained in a practical analysis is given by the product:

$$
L=\prod(2 \pi)^{-1}|M|^{-1 / 2} \exp \left(-\frac{1}{2} \Delta M^{-1} \Delta^{\tau}\right)
$$

Taking the observational variance $\sigma_{\varepsilon_{j}}^{2}$ into account, we can write for the covariance tensor of the residuals:

$$
M=\left(\begin{array}{cc}
\sigma_{\varepsilon_{1}}^{2}+\sum_{i=1}^{3} a_{i 1}^{2} \sigma_{i}^{2} & \sum_{i=1}^{3} a_{i 1} a_{i 2} \sigma_{i}^{2} \\
\sum_{i=1}^{3} a_{i 1} a_{i 2} \sigma_{i}^{2} & \sigma_{\varepsilon_{2}}^{2}+\sum_{i=1}^{3} a_{i 2}^{2} \sigma_{i}^{2}
\end{array}\right)
$$

The residuals of each star depend on the nature of the adopted model for the kinematic properties of the galaxy. Following the conventional practice, we have:

$$
\begin{aligned}
\Delta_{1}=\mu_{\alpha} \cos \delta & -f(X \sin \alpha-Y \cos \alpha)-\omega_{1} \cos \alpha \sin \delta-\omega_{2} \sin \alpha \cos \delta+\omega_{3} \cos \delta \\
\Delta_{2}=\mu_{\delta} & +P\left(\cos 2 l \cos b \cos \phi+\frac{1}{2} \sin 2 l \cos 2 b \sin \phi\right) \\
& -f(X \cos \alpha \sin \delta+Y \sin \alpha \sin \delta-Z \cos \delta)+\omega_{1} \sin \alpha-\omega_{2} \cos \alpha \\
& +P\left(\cos 2 l \cos b \sin \phi-\frac{1}{2} \sin 2 l \sin b \cos \phi\right)
\end{aligned}
$$

where $\left(\mu_{\alpha} \cos \delta, \mu_{\delta}\right)$ are observed proper motions. So the log likelihood function:

$$
l=-\frac{1}{2} \sum\left(\ln |M I|+\Delta M^{-1} \Delta^{\tau}\right)+\text { cons.st. }
$$

is a function of the unknowns $\sigma_{i}, X, Y, Z, \omega_{i}, P,(\mathrm{i}=1,3)$.

Maximum-likelihood estimates of the parameters are those for which the function $-l$ is a minimum. It conld be shown that the covariance matrix of the estimated parameters is the Hessian matrix of $-l$ :

$$
H=-\left(\begin{array}{cccc}
\frac{\partial^{2} l}{\partial x^{2}}, & \frac{\partial^{2} l}{\partial x_{1} \partial x_{2}}, & \ldots, & \frac{\partial^{2} l}{\partial x_{1} \partial x_{n}} \\
\frac{\partial^{2} l}{\partial x_{1} \partial x_{2}}, & \frac{\partial^{2} l}{\partial x_{2}^{2}}, & \ldots, & \frac{\partial^{2} l}{\partial x_{2} \partial x_{n}} \\
\vdots & \vdots & \ldots & \vdots \\
\frac{\partial^{2} l}{\partial x_{1} \partial x_{n}}, & \frac{\partial^{2} l}{\partial x_{2} \partial x_{n}}, & \ldots & \frac{\partial^{2} l}{\partial x_{n}^{2}}
\end{array}\right)
$$

The error of uint weight is:

$$
\sigma_{0}^{2}=-2 l_{0} / N
$$

where $\mathrm{N}$ is the number of degrees of freedom.

\section{RESULTS AND DISCUSSION}

The results for Solar motion $\left(X, Y, Y^{*}\right)$, the components of angular vector $\left(\omega_{1}, \omega_{2}, \omega_{33}\right)$ and (ort's constant $P$ are listed in Table 1 to 3 , including the precessional corrections $\Delta n, \Delta k$ 
Table 1, Results from all usable stars

\begin{tabular}{|c|c|c|c|c|c|c|c|c|c|c|}
\hline MIag. & $10-11$ & $10-13$ & $11-13$ & $8-10$ & $8-11$ & $8-13$ & $8-9$ & $9-10$ & $9-11$ & $9-13$ \\
\hline$\sigma_{0}$ & 123 & 123 & 176 & 167 & 130 & 136 & 116 & 145 & 141 & 120 \\
\hline$\sigma_{1}$ & 772 & 772 & 1374 & 993 & 722 & 672 & 2056 & 1013 & 752 & 682 \\
\hline$\sigma_{2}$ & 673 & 673 & 975 & 843 & 673 & 622 & 1577 & 933 & 693 & 622 \\
\hline$\sigma_{3}$ & 512 & 512 & 724 & 502 & 462 & 452 & 784 & 472 & 462 & 442 \\
\hline 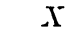 & 03 & 03 & 55 & 73 & 43 & 43 & 116 & 103 & 13 & 23 \\
\hline $\mathrm{Y}$ & -1364 & -1304 & -1026 & -2054 & -1803 & -1713 & -2369 & -2165 & -1684 & -1603 \\
\hline$Z$ & 1242 & 1242 & 1044 & 1583 & 1472 & 1422 & 1835 & 1463 & 1372 & 1342 \\
\hline$\omega_{1}$ & 163 & 163 & 126 & 164 & 143 & 133 & 118 & 384 & 173 & 173 \\
\hline$\omega_{2}$ & -473 & -473 & -385 & -423 & -453 & -443 & -446 & -413 & -493 & -453 \\
\hline$\omega_{3}$ & -402 & -492 & -504 & -383 & -412 & -422 & -355 & -343 & -422 & -442 \\
\hline$\Delta n$ & 503 & 503 & 415 & 453 & 483 & 473 & 466 & 494 & 533 & 493 \\
\hline$\Delta k$ & -413 & -413 & -445 & -303 & -343 & -352 & -290 & -143 & -333 & -353 \\
\hline$P$ & 194 & 194 & 56 & 174 & 20) 3 & 203 & 28 & 434 & 213 & 20) 3 \\
\hline$Q$ & -184 & -184 & -146 & -185 & -164 & -153 & -139 & -445 & -204 & -203 \\
\hline
\end{tabular}

Table 2, Results from star with $\mu<12^{\prime \prime} /$ cy

\begin{tabular}{|rcccccccccc|}
\hline Mag. & $10-11$ & $10-13$ & $11-13$ & $8-10$ & $8-11$ & $8-13$ & $8-0$ & $9-10$ & $0-11$ & $0-13$ \\
\hline$\sigma_{0}$ & 113 & 113 & \multicolumn{1}{c}{110} & \multicolumn{1}{c}{127} & 124 & 122 & \multicolumn{1}{c}{133} & 124 & 121 & 108 \\
\hline$\sigma_{1}$ & 542 & 542 & 953 & 602 & 492 & 472 & 1144 & 682 & 512 & 492 \\
$\sigma_{2}$ & 502 & 502 & 784 & 582 & 492 & 462 & 914 & 612 & 502 & 472 \\
$\sigma_{3}$ & -442 & 442 & 563 & 452 & 422 & 412 & 603 & 462 & 422 & 412 \\
$I^{2}$ & -22 & -22 & 24 & -43 & -42 & -32 & -34 & -53 & -42 & -32 \\
$Y$ & -1083 & -1083 & -825 & -1493 & -1343 & -1282 & -1715 & -1403 & -1263 & -1213 \\
$Z$ & 1042 & 1042 & 923 & 1232 & 1172 & 1142 & 1323 & 1192 & 1142 & 1112 \\
$\omega_{1}$ & 133 & 133 & 94 & 83 & 82 & 82 & 65 & 103 & 102 & 102 \\
$\omega_{2}$ & -442 & -442 & -394 & -422 & -432 & -432 & -404 & -433 & -442 & -442 \\
$\omega_{3}$ & -502 & -502 & -563 & -392 & -422 & -432 & -373 & -392 & -432 & -452 \\
$\Delta n$ & 472 & 472 & 414 & 442 & 452 & 452 & 414 & 453 & 462 & 462 \\
$\Delta k$ & -432 & -432 & -514 & -352 & -382 & -392 & -344 & -343 & -382 & -402 \\
$P$ & 183 & 183 & 74 & 163 & 183 & 182 & 135 & 163 & 183 & 182 \\
$Q$ & -153 & -153 & -105 & -93 & -93 & -93 & -75 & -124 & -123 & -123 \\
\hline
\end{tabular}

Table 3, Results from star with $\mu<6^{\prime \prime} /$ cy

\begin{tabular}{|c|c|c|c|c|c|c|c|c|c|c|}
\hline Iag. & -11 & -13 & $11-13$ & $8-10$ & $8-11$ & $8-13$ & $8-9$ & $9-10$ & $9-11$ & $9-13$ \\
\hline$\sigma_{0}$ & 115 & 115 & 100 & 103 & 103 & 102 & 105 & 113 & 107 & 102 \\
\hline$\sigma_{1}$ & 432 & 432 & 723 & 482 & 391 & 371 & $\overline{923}$ & 12 & 402 & 381 \\
\hline$\sigma_{2}$ & 402 & 402 & 613 & 442 & 391 & 361 & 643 & 472 & 402 & 1 \\
\hline$\sigma_{3}$ & 401 & 1 & 493 & 381 & 381 & 381 & 472 & 392 & 391 & 91 \\
\hline$x$ & -62 & -62 & -13 & 122 & (1) 2 & -102 & -123 & -122 & 102 & -92 \\
\hline$Y$ & -782 & -782 & -624 & 992 & -912 & -882 & 1114 & -953 & -882 & -842 \\
\hline$Z$ & 842 & 42 & 702 & 922 & 901 & 881 & 963 & 90) 2 & 881 & 871 \\
\hline$\omega_{1}$ & 72 & 72 & 33 & 22 & 32 & 42 & -14 & 52 & 52 & 5 \\
\hline$\omega_{2}$ & -442 & -442 & -403 & 432 & -442 & -442 & -413 & -442 & -452 & -442 \\
\hline$\omega$ & -481 & -481 & -552 & -361 & $\begin{array}{lll}-391 & \end{array}$ & -411 & -352 & -362 & -411 & -421 \\
\hline$\Delta n$ & 462 & 462 & 413 & 432 & 452 & 452 & 413 & 452 & 462 & 452 \\
\hline$\Delta k$ & -442 & -442 & -533 & -352 & -372 & .392 & -363 & -332 & -382 & -392 \\
\hline$P$ & $1\rceil 2$ & 172 & 84 & 152 & 162 & 162 & 114 & 153 & 172 & 172 \\
\hline ( & -82 & -82 & -34 & -23 & -32 & -52 & 14 & -63 & -62 & -62 \\
\hline
\end{tabular}



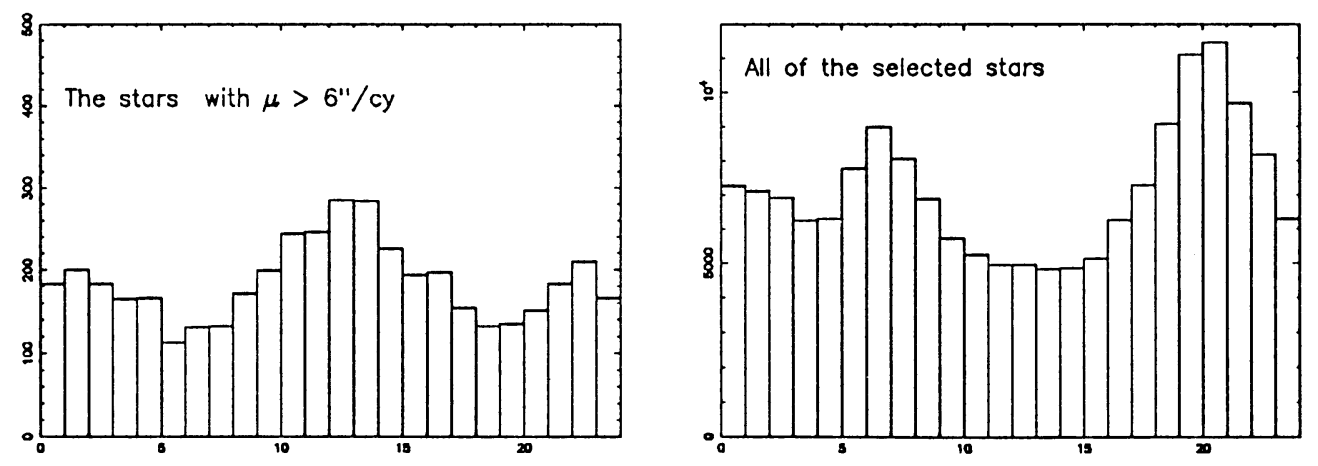

Figure 1, Distribution of AGK3U stars in R. A.
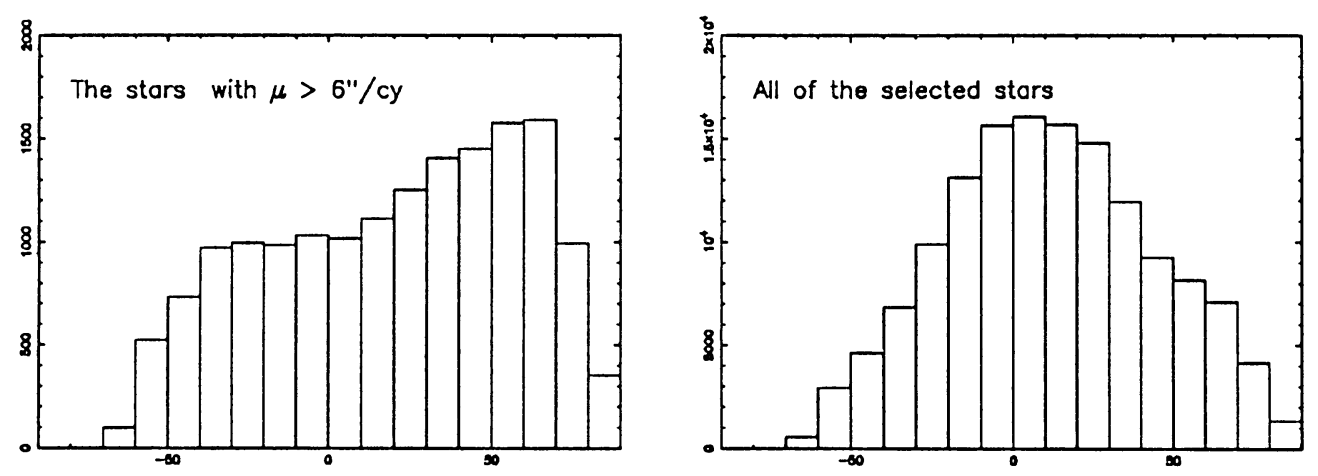

Figure 2. Distribution of AGK3U stars galactic lattitude 
and Oort's constant $Q$ derived from $\left(\omega_{1}, \omega_{2}, \omega_{3}\right)$. The main results can be sumarized as follow:

1), magnitude equation: No siginificant magnitude equation was detected. Only the result of solar motion $(x, y, z)$ varies in different magnitude intervals, and it is resulted from that brighter stars, generally speaking, are nearer to the Sun. But the dependence of solar motion on magnitude interval implies that it might be questionable to use unit parallax factor for all stars.

2), effect of large proper motions: There is significant difference in the results of stellar kinematic parameters in the solutions of all stars and with exclusion of large proper motions. This is different from the conclusion of Asteriadis(107T). In order to explain this disagreement, we plotted the $\alpha$ distribution and $b$ distribution of all of the selected stars and large proper motion stars (Figure 1 and 2). From these distributions, it can be concluded that the large proper motion are maily caused by solar motion.

3), precessional corrections: The results for precessional corrections are very stallele in different solutions. And $\Delta n=0^{\prime \prime} .47 \pm 0^{\prime \prime} .03 / \mathrm{cy}, \Delta k=-0^{\prime \prime} .35 \pm 0^{\prime \prime} .02 / \mathrm{cy}$ resluting from all of the selected stars are in good agreement with the results obtained by by Asteridis(19Ti) from AGK3.

4), galactic rotation constants: The results for Oort's constants are confusing. Oort's constants become quit small after the exclusion of large proper motions. Since the distributions shown in figure 1 and 2 express that the large proper motions contribute very little to the determination of Oort's constants, the dependence of Oort's constants on the fast. stars must be resulted from the disadvantage that all of the stars are distributed in north sky.

\section{REFERENCES}

Asteriarlis, G., 10TT, Astron. Astrophys., vol. 56; 25

Bucciarelli, B., 1992, Astron. J., vol. 103; 1689

Dieckvoss, W., 1967, Astron. Na.chr., vol. 290; 141

Milalas, D. and Binncy, J.J., 1982, Galactic Astronomy, San Francisico:Freeman Murray, C.A., 1983, Vectorial Astronomy, Adam Higer Ltd.

Wei, X., Vistas in Astron., vol. 31; 677 